\title{
Involuntary attention and identification accuracy
}

\author{
WILLIAM PRINZMETAL, SAM PARK, and ROSALIE GARRETT \\ University of California, Berkeley, California
}

\begin{abstract}
Using the spatial cuing paradigm, Prinzmetal, McCool, and Park (2005) made the distinction between voluntary and involuntary attention. They claimed that although accuracy was affected by an informative spatial cue (which controls voluntary attention), it was not affected by a noninformative cue (which controls involuntary attention). We reevaluate two reports that assert that noninformative spatial cues affect accuracy. Dufour (1999) reported that a noninformative auditory cue enhanced visual identification in a conjunction search task. Klein and Dick (2002) reported that, in an RSVP task with visual cues, the cue also enhanced accuracy at short stimulus onset asynchronies. We found that Dufour's results were due to overt orienting (eye movements) rather than to covert attention. The results of Klein and Dick were due either to location uncertainty or to a confounding of the order of stimulus presentation and condition.
\end{abstract}

It has been known for over 100 years that one can fixate one's eyes on one location yet attend to another location (Helmholtz, 1896; James, 1890; Wundt, 1912). Historically, it has been suggested that there are two varieties of this kind of attention. For example, Wundt (1902) commented that attention could be "involuntary" or "voluntary." He thought that these two forms of attention differed only in complexity, but that they were mediated by the same mechanisms. A variety of distinctions that correspond more or less to Wundt's (1902) voluntary and involuntary forms of attention has been made, including those between endogenous attention and exogenous attention, push cues and pull cues, and central cues and peripheral cues (see, e.g., Jonides, 1976; Klein \& Shore, 2000; Posner, 1980). Most experimenters investigating this distinction have used the spatial cuing task developed by Michael Posner and his colleagues (Posner, 1978, 1980; Posner, Snyder, \& Davidson, 1980). In this paradigm, observers engage in either detection or identification of a peripherally presented stimulus. However, before the stimulus appears, observers are precued to its possible location. On valid trials, the cue indicates the target location; on invalid trials, the cue indicates a nontarget location.

Jonides $(1976,1980)$ investigated the distinction between voluntary and involuntary attention in this paradigm, using a very simple manipulation. To investigate voluntary attention, the cue was informative or predictive

We thank Atusa Heidarian, Artem Violentyev, Paul Park, and Vivian Wang for their help in collecting the data for these experiments, and Michael Esterman and Lynn Robertson for their comments on the manuscript. We thank André Dufour and Charles Spence for their valuable insights on cross-modal cuing, and Steve Luck for his advice on the manuscript. Finally, we express our gratitude to Ray Klein, without whose assistance Experiments 3 and 4 would not have been possible. Correspondence concerning this article should be addressed to W. Prinzmetal, Psychology Department, University of California, Berkeley, CA 94720-1650 (e-mail: wprinz@socrates.berkeley.edu). of the target location. Thus, on a majority of trials the cue indicated the target location. Under these circumstances, it is advantageous for participants to voluntarily allocate attention to the cued location. To investigate involuntary attention, Jonides $(1976,1980)$ used cues that were nonpredictive of or noninformative about the target location. Thus, the cue was random with respect to the target location. With random cues, there is no reason for participants to allocate their attention to the cued location, and any effect that the cues have is involuntary. Both informative cues and automatic cues affect reaction time (RT): Observers are faster at detecting or classifying a stimulus in the cued location than in an uncued location (Jonides, 1976, 1980).

There is some evidence that voluntary and involuntary cues differ in a number of respects (see, e.g., Jonides, 1981; Juola, Koshino, \& Warner, 1995; Müller \& Rabbitt, 1989; Posner, Cohen, \& Rafal, 1982; Spence \& Driver, 1994; Warner, Juola, \& Koshino, 1990). For example, as the interval between the onset of the cue and the onset of the target (i.e., stimulus onset asynchrony, or SOA) increases, the facilitation at the cued location becomes inhibition (see, e.g., Posner \& Cohen, 1984). This effect is called inhibition of return (IOR). IOR occurs only with involuntary attention (controlled by noninformative cues) and not with voluntary attention (controlled by informative cues; Posner \& Cohen, 1984; Richard, Wright, \& Ward, 2003).

It is clear that voluntary and involuntary attention differ in how they are summoned. For example, as was described above, involuntary attention has its maximum effect with short cue-target SOAs, whereas voluntary attention has its maximum effect at longer SOAs. However, the question remains as to whether voluntary and involuntary attention affect the same processes once selection has taken place. Recently, Prinzmetal, McCool, and Park (2005) have proposed that voluntary and involuntary attention affect different processes subsequent to selection. They 
claim that voluntary attention enhances the perceptual representation, so that, in an experiment designed around accuracy, observers will be more accurate on valid trials than on invalid trials. Involuntary attention does not affect the perceptual representation. Instead, it reflects a process they call "channel selection." Channel selection involves a decision about which location contains the target or which location requires a response - that is, which location is the relevant "channel." In a sense, the Stroop task involves a form of channel selection wherein the channels are the word and the color. In the Stroop task, the conflict concerns which response to make in that the word incites one response but the color another. In the spatial cuing paradigm, on invalid trials, the cue provokes the observer to respond to one location, but the target is in another location. As for the Stroop task, it was hypothesized that noninformative cues would affect the speed of channel selection but not the perceptual representation. Thus, noninformative cues affect performance in RT experiments but not in accuracy experiments. ${ }^{1}$

A critical test of the nature of voluntary attention and involuntary attention is whether noninformative cues affect performance in experiments in which only accuracy is emphasized and there is no emphasis on speed of processing. Prinzmetal et al. (2005) reviewed several experiments by authors who claimed to find effects on response accuracy with nonpredictive cues. They found that the various results could be accounted for by one of the following four factors: (1) The experiments were not truly accuracy experiments, but there was pressure to respond quickly as well as accurately (e.g., Cheal \& Chastain, 1999; McDonald, Teder-Sälejärvi, \& Hillyard, 2000). (2) Eye movements were not monitored (e.g., Dufour, 1999). (3) Observers were unable to determine with certainty where the target was located (i.e., they demonstrated location uncertainty; see Luck, Hillyard, Mouloua, \& Hawkins, 1996; Prinzmetal, Nwachuku, Bodanski, Blumenfeld, \& Shimizu, 1997, Experiments 5 and 6; Shiu \& Pashler, 1994). (4) There were factors that were confounded with the cue validity manipulation (valid vs. invalid trials). For example, Handy, Jha, and Mangun (1999) masked only the location containing the target. A consequence of this is that on valid trials one screen location contained stimulus material, whereas on invalid trials two locations contained stimulus material. Thus, the number of locations containing stimulus material was confounded with the type of cue (see Prinzmetal et al., 2005).

Prinzmetal et al. (2005) demonstrated that both informative and noninformative cues affected performance in RT experiments, but only informative cues (i.e., those that control voluntary attention) affected performance in experiments designed around accuracy. Their experiments involved four different versions of the spatial cuing paradigm, including those making use of (1) visual cues and a letter discrimination task, (2) auditory cues and a visual line orientation task, (3) visual cues and a face discrimination task, and (4) visual cues and a visual line orientation task. In these experiments, a range of temporal parameters (e.g., different SOAs, different exposure durations) was tested, and the results were consistent. In experiments designed around RT, both informative and noninformative cues affected performance: Observers were faster on valid trials than on invalid trials. However, in the experiments designed around accuracy, only informative cues affected performance. In seven different experiments concerning involuntary attention, there was no effect on accuracy.

In the present work, we closely examine two reports that assert that noninformative cues affect accuracy. In these studies, observers were more accurate on valid than on invalid trials (at short cue-stimulus SOAs). Dufour (1999) used a cross-modal cuing paradigm (involving auditory cues and visual targets) and found that accuracy was higher when the stimulus appeared near the location of a noninformative auditory cue than when it appeared far from the source of the auditory cue. Klein and Dick (2002), using a rapid serial visual presentation (RSVP) task, reported that noninformative visual cues affect performance in a similar manner. Our goal was to see whether we could account for the discrepancy between the results of Prinzmetal et al. (2005) on the one hand and those of Dufour (1999) and Klein and Dick (2002) on the other.

In Experiments 1 and 2, we examine the results of Dufour (1999). Experiment 1A was a replication of Dufour's experiment, and Experiment 1B was identical to Experiment 1A except that we controlled the observers' eye movements. Experiments 2A and 2B were identical to Experiments $1 \mathrm{~A}$ and $1 \mathrm{~B}$, respectively, except that the stimuli consisted of black letters on a white background. Experiment 3 was an exact replication of the experiment of Klein and Dick (2002). When running this experiment, we noticed two problems and corrected them in Experiment 4.

To summarize our conclusions, we found that Dufour's (1999) results could be accounted for by eye movements. Klein and Dick's (2002) results may have been due to a confounding of the order of stimulus presentation, since all of their observers were tested with the stimuli in the same order. Alternatively, their results could have been a consequence of the fact that observers were not given feedback and may have believed that the target was more likely to appear in the cued stream. When we eliminated these factors (Experiment 4), the cue had no effect on accuracy.

\section{EXPERIMENTS 1A AND 1B}

Dufour (1999, Experiment 1) had observers perform a very difficult conjunction search task (Treisman \& Gelade, 1980). The task was to indicate the orientation of the letter $\mathrm{T}$ (upright, tilted left, tilted right, or upside down) when it was surrounded by other Ts in different orientations. Figure 1 is an example of the stimuli used both by Dufour and in our Experiments 1 and 2. However, the stimuli used by Dufour and in our Experiment 1 were white on a black background. In our Experiment 2, the stimuli were black on a white background (as in Figure 1). 


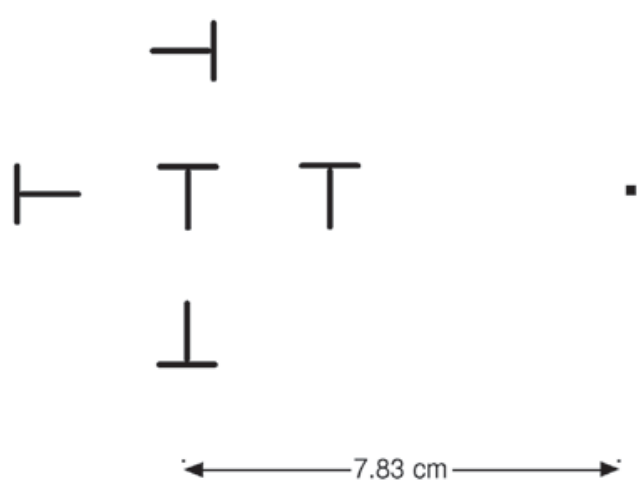

Figure 1. A sample stimulus used in Experiments 1 and 2. In Experiment 1, the stimuli were white letters on a black background, whereas in Experiment 2 the stimuli were black letters on a white background. The figure is drawn to scale.

In both Dufour's (1999) experiment and our own, the visual stimulus was preceded by a lateralized tone for $100 \mathrm{msec}$. The tone was not predictive of the target location. Dufour found that identification performance was approximately $7.5 \%$ more accurate when the tone and the target were on the same side (i.e., on valid trials) than when they were on opposite sides (i.e., on invalid trials). These results counter those of Prinzmetal et al. (2005), who found no difference in performance between valid and invalid trials if the cue was not predictive. One possible difference between the experiments is that Dufour used a complex discrimination task that involved feature integration, whereas the cross-modal experiments of Prinzmetal et al. (2005) involved a simple line orientation discrimination that did not necessarily require attention. (Note, however, that Prinzmetal et al., 2005, did find an effect of attention when the cue was informative.) Accuracy in tasks involving feature integration is affected by informative spatial cues (see, e.g., Prinzmetal, Presti, \& Posner, 1986). At issue is whether involuntary attention affects feature integration.

Another possibility, suggested by Spence (2001), is that Dufour's (1999) results may have been due to eye movements. Dufour did not monitor eye movements because he felt that the time between the onset of the auditory cue and the offset of the target $(160 \mathrm{msec})$ was too short to allow participants to complete a saccadic eye movement. As we will argue in Experiment 2, this assumption is probably wrong. Furthermore, the stimuli were white letters on a black CRT in a dimly lit room, and they were not masked. Hence, there may have been a fading trace on the CRT screen and additional time for participants to move their eyes. On valid trials, an eye movement to near the target location would have increased accuracy. In tasks that involved feature integration, such as that of Dufour, the negative effect of crowding items with similar features increases dramatically with eccentricity (Pelli, Palomares, \& Majaj, 2004; see also Banks, Larson, \& Prinzmetal, 1979, and Bouma, 1970). Thus, eye movements toward the cued side would increase accuracy on valid trials and decrease performance on invalid trials. The cue could also have another, more subtle effect on overt orienting. It may be that on both valid and invalid trials the observers moved their eyes to the target location, but the latency of such eye movements was shorter on valid than on invalid trials. Fischer and Weber (1998) found that, with noninformative spatial cues, saccadic latency was dramatically shorter with valid cues than with invalid cues. Hence, the target would be more likely to still be visible on valid trials than on invalid trials

To test the possibility that Dufour's (1999) results may have been due to eye movements, we ran two experiments that were similar to his Experiment 1 in terms of stimuli and procedure. In both experiments, we monitored eye movements. However, in Experiment 1A we simply monitored eye movements and did not actively control or suppress them. In Experiment 1B, we actively discouraged eye movements by instructing the observers to not move their eyes and giving them feedback whenever they did move their eyes.

\section{Method}

Observers. A different group of 15 observers, 18-25 years of age, was tested in each experiment. In all of the experiments reported in this article, the observers were recruited from the subject pool of the psychology department of the University of California at Berkeley and were given course credit in exchange for their participation.

Procedure. The observers' task was to indicate the orientation of a briefly presented $\mathrm{T}$ surrounded by distractor Ts in four different orientations (see Figure 1). The sequence of events in each trial was as follows. A $440-\mathrm{Hz}$ sine wave tone was played for $100 \mathrm{msec}$ through two speakers located either to the left or to the right of the observer. Forty milliseconds after the onset of the tone, the letter array was presented in either the left or the right visual field for $120 \mathrm{msec}$. The observers responded by pressing one of four buttons on a button box. The observers were urged to take their time and to be as accurate as possible.

Each observer received two blocks of practice trials followed by five blocks of test trials. Each block included 48 trials; on half of the trials, the tone was emitted from speakers on the same side as the visual stimulus (valid trials), and on the other half the tone was emitted from speakers on the side opposite that of the visual stimulus (invalid trials). The observers received feedback at the end of each block. In Experiment 1A, eye movements were monitored but no explicit mention of eye movements was made. In Experiment 1B, the observers were told not to move their eyes. When an eye movement was detected, the computer emitted a sound somewhat like that of a foghorn.

We monitored eye movements with a video camera located approximately $15 \mathrm{~cm}$ from the observer's left eye. The image of the observer's eye was presented on a monitor and magnified by about four times. The experimenter monitored eye movements and signaled the computer whenever an eye movement was suspected. In independent calibrations, we found that we could detect an eye movement to the target location with a hit rate of over .99 and a false alarm rate of under 01

Stimuli. The stimuli were presented on a 17 -in. Apple monitor. The viewing distance was $58 \mathrm{~cm}$, and a chinrest was used to minimize head movements. The stimuli were drawn as shown in Figure 1, except that they consisted of white letters on a black background. The distance from the fixation point to the center of the target $\mathrm{T}$ subtended $7.6^{\circ}$ of visual angle. Dufour (1999) had used four target- 
distractor distances (from $1.3^{\circ}$ to $2.11^{\circ}$ ), but we used only the one shown in Figure $1\left(1.6^{\circ}\right)$. Note that Dufour's cuing effect did not significantly interact with target-distractor distance.

On each trial, the orientation of the target $T$ was randomly determined. The distractor Ts were always presented in all four orientations, but in different randomly determined locations on each trial.

The cue was a $100-\mathrm{msec}, 440-\mathrm{Hz}$ sine wave tone with a sound pressure level of approximately $88 \mathrm{~dB}$. The sound was delivered via four Cambridge Soundworks speakers, two on the observer's left and two on his or her right. One speaker was located on each side of the monitor ( $23 \mathrm{~cm}$ from its center). The other two were located on a table, about $16 \mathrm{~cm}$ in front of the observer and approximately $34 \mathrm{~cm}$ to either side of the observer's line of sight. The cue was presented by a tone in either the left pair or the right pair of speakers. ${ }^{2}$

\section{Results and Discussion}

The results of Experiment 1A nicely replicated those of Dufour (1999). The observers were more accurate on valid than on invalid trials ( $86.1 \%$ vs. $77.8 \%$, respectively), and this difference was reliable $[t(14)=3.61, p<.01]$. Note that in Experiment 1A we monitored eye movements but did not mention anything about eye movements to the observers. The analysis includes all the trials, regardless of whether or not eye movements were detected. ${ }^{3}$

The results of Experiment 1B, in which we actively controlled eye movements, were entirely different. The difference between valid and invalid trials was extremely small. The percentages correct for valid and invalid trials were $69.7 \%$ and $68.5 \%$, respectively $[t(14)=1.06$, n.s.]. This analysis excludes the $3 \%$ of trials on which eye movements were made.

Note that accuracy in Experiment 1A was higher than that in Experiment 1B. This result is consistent with the fact that the observers could move their eyes and take advantage of screen persistence. Our conclusion is that the results of Dufour's (1999) experiment could have been due to uncontrolled eye movements.

\section{EXPERIMENTS 2A AND 2B}

Dufour (1999) assumed that as long as the time between the onset of the cue and the offset of the target was $160 \mathrm{msec}$, observers would not have sufficient time to move their eyes. In Experiment 1, the time between the onset of the cue and the complete disappearance of the target was probably longer than $160 \mathrm{msec}$ due to a slowly fading phosphor trace. However, Dufour's assumption that $160 \mathrm{msec}$ sufficed to preclude eye movements may be incorrect. It is based on the observation that in reading of connected discourse (see, e.g., Sekuler \& Blake, 1985, p. 267) or free viewing (Fischer \& Weber, 1993), humans make three to five saccades per second.

It may be that the saccade latencies measured during free viewing or reading of connected discourse do not reflect the speed of saccades with single simple saccadic targets, such as those used in the present experiment. Dufour's (1999) experiment is similar to saccade latency experiments in which a single target is presented in the periphery. In these experiments, the observer's task is to move his or her eyes to a peripheral target. Many of these experiments are similar to Dufour's experiment in that a very simple target is presented in one of only a few peripheral locations. Under these circumstances, saccadic latency (the time from onset of the target to the completed saccade) can be as short as $50 \mathrm{msec}$ and often averages 100-150 msec (see, e.g., Fischer \& Ramsperger, 1984; Fischer \& Weber, 1993; Gezeck, Fischer, \& Timmer, 1997; Madelain, Harwood, Krauzlis, \& Wallman, 2004; Weber, Biscaldi, \& Fischer, 1995). ${ }^{4}$ The conditions of Dufour's experiment (i.e., a few known target locations and temporal certainty) are just those that promote fast saccades (Cameron, 1995; Harwood, Madelain, Krauzlis, \& Wallman, 2003; Jüttner \& Wolf, 1994). However, even if the average latency is $150 \mathrm{msec}$, because the distribution of saccadic latencies is positively skewed, more than half of the saccades would have been completed by $160 \mathrm{msec}$. Because saccades are faster following a valid cue than following an invalid cue (Fischer \& Weber, 1998), the probability of completing a saccade is greater on valid trials than on invalid trials. Thus, even without the persistent phosphor trace with white stimuli on a black background, $160 \mathrm{msec}$ might not have been long enough to preclude eye movements affecting performance. In Experiments $2 \mathrm{~A}$ and 2B, we tested this hypothesis by reversing stimulus brightness, presenting black letters on a white background.

\section{Method}

In Experiment 2A, we allowed eye movements, and in Experiment $2 \mathrm{~B}$ we discouraged eye movements in the same manner as in Experiment 1B. The experiments differed from Experiments $1 \mathrm{~A}$ and 1B, respectively, only in the following respects: (1) The stimuli consisted of black letters on a white background; (2) eye movements were not monitored in Experiment 2A, but they were monitored in Experiment 2B; and (3) 18 observers participated in each experiment.

\section{Results}

The results of Experiment 2A were similar to those of Dufour (1999) and of our Experiment 1A. When eye movements were permitted and not monitored, the observers were significantly more accurate on valid trials than on invalid trials $[70.1 \%$ vs. $63.7 \%$, respectively; $t(17)=$ $2.69, p<.05]$.

In Experiment 2B, 1 participant was eliminated from the analysis because of an excessive number of eye movements. This observer made eye movements on $17.9 \%$ of the trials, whereas the other observers averaged eye movements on only $1.7 \%$ of the trials. For the remaining 17 observers, there was a slight advantage for valid trials over invalid trials (67.0\% vs. $65.6 \%$, respectively), but this difference was not significant $[t(16)=1.17]$.

We conducted a post hoc meta-analysis with stimulus color (black vs. white) and eye movement (permitted vs. not permitted) as between-subjects factors and cue validity as a within-subjects factor. In this overall analysis, there was a significant interaction of cue type and eye movement $[F(1,62)=10.01, p<.001]$. Hence, we conducted separate simple-effects tests for the movement-permitted and movement-not-permitted groups. The former group had a significant effect of cue type $[F(1,31)=19.41, p<$ 
$.001]$, but for the latter group cue type was not significant $[F(1,30)=2.47]$.

\section{Discussion}

We do not take a position on whether or not a specific interval between the onset of the cue and the offset of the target is sufficient to preclude eye movements. In order to preclude eye movements, the most efficient method is to monitor them. Fortunately, the method that we used is both inexpensive and accurate. However, although we can reliably determine whether or not observers moved their eyes, we cannot determine precisely when and where they moved their eyes.

Our conclusion is that Dufour's (1999) results probably reflected overt orienting. When we did not prevent eye movements, we replicated Dufour's results; when we monitored and discouraged eye movements, we did not.

One could argue that actively suppressing eye movements might negate the effect of involuntary attention. There might be a connection between automatic attention and eye movements (see, e.g., Rafal, Calabresi, Brennan, $\&$ Sciolto, 1989). It is possible that instructions not to move one's eyes somehow overrode the automatic attention system. We know of only one test of this hypothesis. Prinzmetal et al. (2005) used auditory cues (white noise) in a task in which participants had to judge whether a line was horizontal or vertical. The stimuli were masked, and the SOA was either 50 or $100 \mathrm{msec}$ long. Prinzmetal et al. (2005) ran both accuracy and RT versions of this task. There were both predictive and nonpredictive cue versions, but only the nonpredictive version (involving involuntary attention) will be discussed here. In the RT experiment, the lines were long and the observers were nearly $100 \%$ correct. In the accuracy experiments, the observers were urged to take their time and to be as accurate as possible. The lines were shortened until the observers were $75 \%-80 \%$ correct. When eye movements were monitored and prevented, in the RT experiment the observers were significantly faster with valid cues than with invalid cues. However, in the accuracy experiment, cue had no effect. Thus, prevention of eye movements did not eliminate the RT effect. Since SOA did not significantly interact with the cue in any of these experiments, Prinzmetal et al. (2005) ran an additional accuracy experiment with only the 50-msec SOA and permitting eye movements. There was still no difference in accuracy between valid and invalid trials. Thus, allowing eye movements did not seem to alter the results of these experiments.

The issue of whether or not discouraging eye movements can eliminate an attention effect that does not depend on eye movements is a tricky one and deserves further investigation. In most spatial cuing experiments, investigators either discourage eye movements or assume that observers are not making them (which might be incorrect). Nevertheless, Prinzmetal et al. (2005) found that in an RT experiment, when they discouraged eye movements, automatic attention affected performance, but in an accuracy experiment, the cue had no effect on performance even when they allowed eye movements. ${ }^{5}$
Experiments 1 and 2 suggest that Dufour's (1999) results were probably due to uncontrolled eye movements. In Experiments 3 and 4, we examine a report by Klein and Dick (2002) that a noninformative spatial cue affected accuracy. They also did not control for eye movements, but we discovered other problems in their experiment; when these were corrected, their effects were eliminated.

\section{EXPERIMENT 3}

Klein and Dick (2002) reported that with noninformative spatial cues, observers were more accurate at the cued location at short cue-target intervals and less accurate at the cued location at long cue-target intervals. These results are also an apparent exception to the findings of Prinzmetal et al. (2005). The experiment of Klein and Dick was unique in several respects. The target stimulus, a digit (2-9), was embedded in one of two simultaneous RSVP streams of letters (see Figure 2). The task was to report the digit. Thus, the task involved complex pattern discrimination and the displays were dynamic. Both of these attributes would seem to require attention.

In Klein and Dick's (2002) experiment, each in a series of 15 frames consisted of three black boxes on a gray background (see Figure 2). The observers were instructed to fixate on the center box. The two RSVP streams consisted of randomly selected letters (excluding B, I, and O) presented in the two peripheral boxes. Each letter was presented for $160 \mathrm{msec}$. There were 15 frames in each stream of characters. The target numeral (2-9) was embedded within one of the streams at frame positions from 6 to 12 . The cue consisted of brightening of one or both of the peripheral boxes. There were four cue conditions: (1) cue in the same stream as the target, (2) cue in the stream opposite that of the target, (3) cue in both peripheral boxes, and (4) cue in the center box only. The latter two conditions were control conditions, and no explicit predictions were made about them. The 6th frame was always the cued frame. Thus, the cue could be simultaneous with the target or could precede the target by 1 to 6 frames. Hence, there were 4 cue types $\times$ 7 target-cue delays, resulting in 28 unique conditions.

The results for the two main conditions are shown in Figure 3 (top panel). When the cue was in the same stream as the target (i.e., valid), their observers were more accurate for the cued frame and for the first few frames following the cue. However, when the target followed the cue by four frames, the results were reversed: The observers were more accurate when the target was in the opposite stream (i.e., when an IOR was present).

Klein and Dick's (2002) results may indicate that if one wants to reveal attention effects in accuracy with a nonpredictive cue (which controls involuntary attention), a dynamic loading of the visual system (as occurs in an RSVP task) is necessary. It may be that performance with an RSVP task is fundamentally different from performance when a single stimulus is presented.

We thought it would be worthwhile to replicate Klein and Dick (2002). Experiment 3 was an exact replication, using the same software (kindly provided to us by 


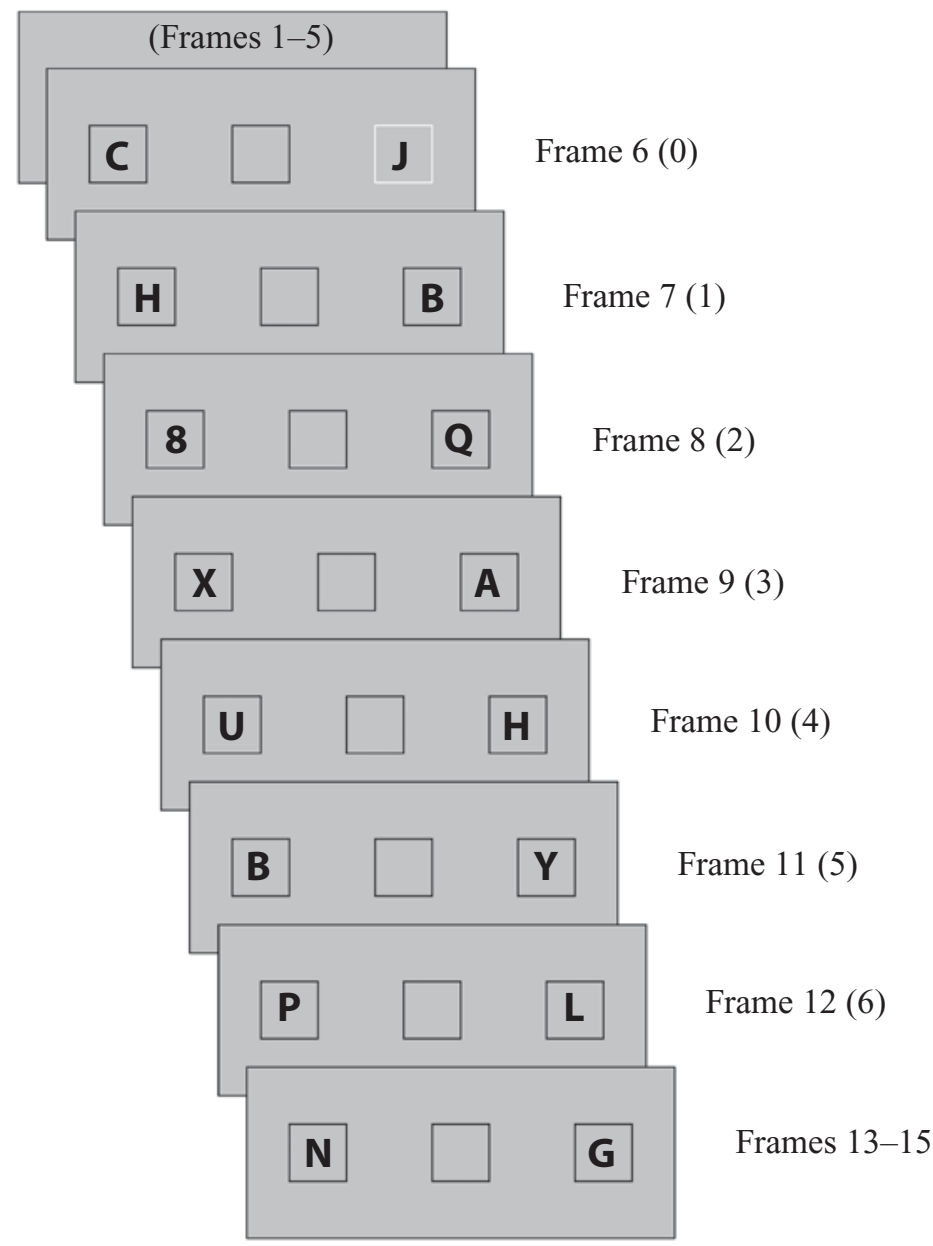

Figure 2. A sample stimulus used by Klein and Dick (2002) and in the replication of their experiment (Experiment 3). The cued box was always in the 6th of 15 frames. The number in parentheses is the lag between the onset of the cue and the target (a digit). The trial illustrated is an opposite-streamcued trial with a cue-target lag of 2 .

Raymond Klein) presented on the same type of monitor (Apple MultiSync monitor set at $75 \mathrm{~Hz}$ ).

While running the experiment, we discovered two problems in Klein and Dick's (2002) experiment. The first problem was a confounding of the order of presentation and the stimulus conditions. We discovered, using the software provided to us, that each participant has been run with the stimuli in the same order. The second problem was that there was a great deal of spatial uncertainty as to the target's location. After having run the experiment ourselves, it occurred to us that often there seemed to be more than one digit. A bias to report the digit that was spatially and temporarily adjacent to the cue could account for the results obtained. Both of these problems will be discussed in detail in Experiment 4.

\section{Method}

Observers. Twenty undergraduates at the University of California at Berkeley participated in exchange for course credit.
Procedure. Experiment 3 was an exact replication of Klein and Dick's (2002) experiment. We used the same software, type of monitor, refresh rate $(75 \mathrm{~Hz})$, and screen resolution $(832 \times 624)$ as Klein and Dick had. Some additional details about Experiment 3 that were not mentioned above or by Klein and Dick are the following. Each trial was initiated by pressing the space bar on the computer. Each of the first 14 frames (see Figure 2) was presented for $160 \mathrm{msec}$. The 15 th frame remained in view until the observer responded. As we mentioned above, the cued frame was always the 6th frame. After one block of 28 practice trials, each observer was tested on 448 trials, or 16 trials per condition. The experimental trials were not broken into blocks, and there was no feedback.

Klein and Dick (2002) did not monitor eye movements because their observers had to monitor two bilateral streams of characters simultaneously, and an eye movement to one stream would have caused the observer to miss a target in the other stream. Because Klein and Dick did not monitor eye movements, we also did not monitor them.

Stimuli. The stimuli were drawn in charcoal in 24-point type. Our observers viewed the displays from a distance of approximately $60 \mathrm{~cm}$. Klein and Dick (2002) did not use a chinrest, so we also did not use a chinrest. Therefore, the exact size of the stimuli, in terms of degrees of visual angle, could not be determined. However, the 

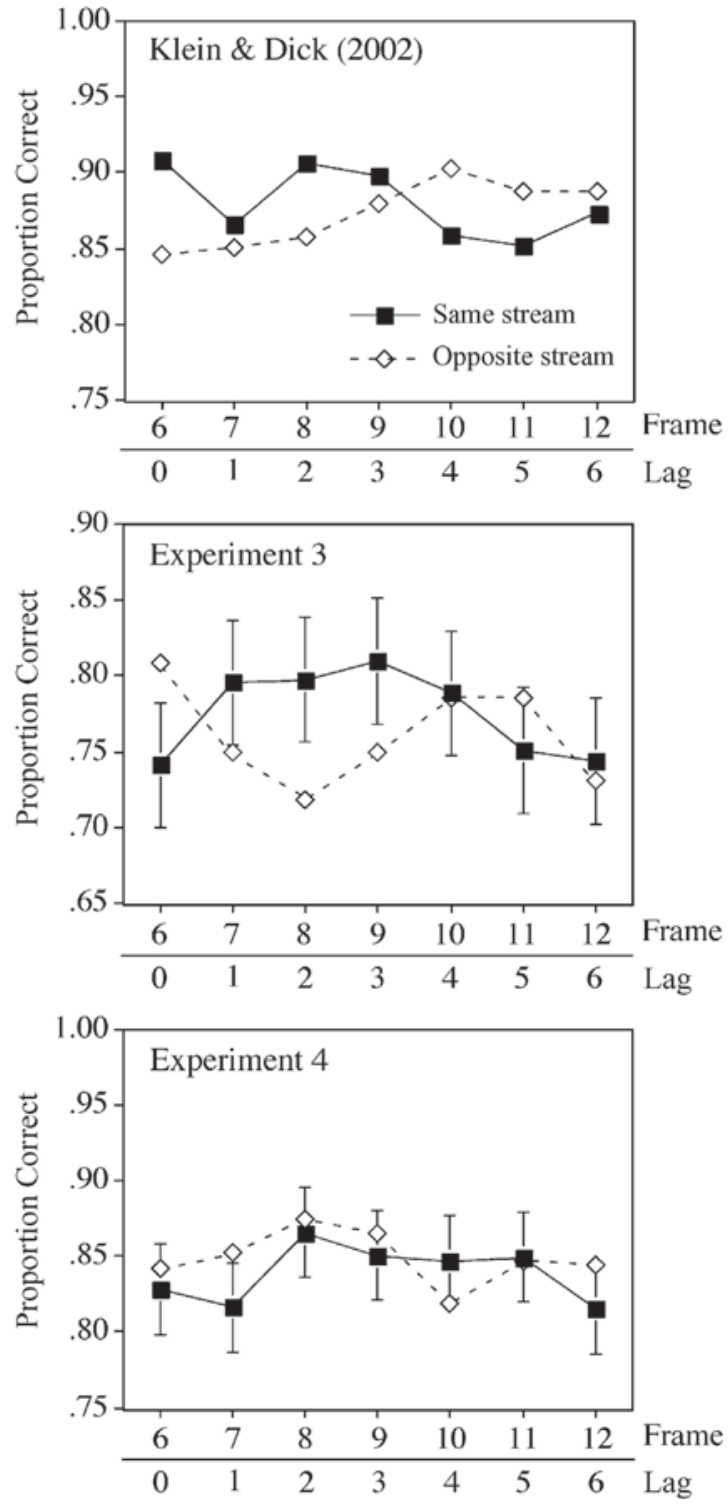

Figure 3. Top panel: The results reported by Klein and Dick (2002). At short cue-target lags, performance was better with the cue and the target in the same stream (i.e., on the same side), but at long cue-target lags this effect was reversed (i.e., an inhibition of return was present). Middle panel: The results of Experiment 3, an exact replication of Klein and Dick. Bottom panel: The results of Experiment 4, a replication of Klein and Dick but with a random order of presentation for each participant and feedback after each trial.

three squares were approximately $1.3 \mathrm{~cm}$ on each side, and the distance between the inside edges of the two outer squares was $7.5 \mathrm{~cm}$. The gray of the background was slightly brighter than the mid-level gray of the monitor (brightness level $=152$, where black $=0$ and white $=255$ ). The target could be simultaneous with the cue (sixth frame) or follow the cue by up to six frames. The 28 practice trials and 448 experimental trials took about $50 \mathrm{~min}$ to complete.

\section{Results and Discussion}

There was an overall significant interaction between cue condition and frame $[F(18,342)=2.49, p<.01]$.
Considering only the critical cue conditions (cue in same stream vs. cue in other stream), there was also a significant interaction between cue condition and frame $[F(6,114)=2.96, p<.01]$. The results of the two critical conditions (same- and opposite-stream cues) are shown in Figure 3 (middle panel). The 95\% confidence intervals were calculated using the method described by Loftus and Masson (1994). These results unaccountably differ in a few respects from the original results of Klein and Dick (2002). Most notably, there was no IOR. The shapes of the functions were also slightly different (see Figure 3). Importantly, however, the main results did replicate those of Klein and Dick: For the first few frames following the cue, the observers were more accurate when the cue was in the same stream of characters as the target (valid trials) than when the cue was in the opposite stream (invalid trials). In our exact replication of Klein and Dick, the facilitation began slightly later and lasted slightly longer than that found in the original experiment. However, using the same computer program and a similar monitor, we replicated their findings of a facilitatory effect of the cue on accuracy.

While running this experiment, however, we discovered that it had two aspects that made us question the results. We investigated these factors in Experiment 4.

\section{EXPERIMENT 4}

While analyzing the data from Experiment 3, we discovered that all of the observers were run with the stimuli in exactly the same order. Klein (personal communication, November 2002) confirmed that "most of the participants were run with the stimuli in the order" of the software they provided to us. Klein and Dick (2002) ran 35 of their 40 participants with the stimuli in the same random order. 6

To determine whether the results of Klein and Dick (2002) could have been caused by the order of stimulus presentation, all trials were numbered (from 1 to 448) and the average ordinal position for each of the 28 conditions was calculated. The average ordinal position of the samestream cued trials over lags $1-4$ is $230(M=221)$. For the other-stream cued trials, the average over the same lags is $209(M=199)$. Thus, on average, the same-stream cued trials came later in the experiment than the otherstream cued trials. If one assumes that the participants improved with practice (and they did in this experiment- see below), it is possible that the order could account for the results. It seemed to us that before any firm conclusions could be drawn about Klein and Dick's results, the experiment needed to be replicated with the stimuli in a different random order for each observer.

The second problem with Klein and Dick's (2002) experiment was that there was a great deal of spatial (and temporal) uncertainty regarding the target's location. Effects on accuracy in the spatial cuing paradigm can occur because the observer is not certain which location contains the target (see Luck et al., 1996; Luck \& Thomas, 1999; Prinzmetal et al., 1997, Experiments 5 and 6; Shiu 
$\&$ Pashler, 1994). In the present paradigm, location uncertainty could affect performance in the following manner. One sometimes perceives two digits on a trial (e.g., an 8 on the left and a 6 on the right) even though, of course, there is always only one. (In the thousands of trials we ran in this experiment, we encountered this phenomenon, and some of our participants have made the same observation.) Suppose one is biased to respond with the digit that is in close spatial and temporal proximity to the cue. Such a bias would result in higher accuracy when the target was in close spatial/temporal proximity to the cue because the participant would be responding to the true target. Performance on trials in which the true target was far from the cue would suffer, since the participant would be responding to a phantom digit. Since Klein and Dick did not provide feedback, there was no opportunity to correct biases such as these. In the present replication, the participants were given trial-by-trial feedback.

In Klein and Dick's (2002) experiment, there can be temporal as well as location uncertainty, and this may explain an anomalous finding. For example, the participant might perceive two digits in the same stream. If the participant was biased to believe that the digit that was closest in time to the cue event was the target, said bias would boost performance when the target was in fact close in time to the cue, but it would hurt performance when the target was far in time from the cue. Without trial-by-trial feedback, there would be no way for this bias to be corrected. This would explain the following anomalous finding. In one of Klein and Dick's control conditions, both peripheral boxes were cued. The results in this condition were nearly identical to those of the same-side condition shown in Figure 3 (top panel). There is no attentional explanation for why cuing both sides would lead to the same results as cuing one side. Our interpretation of this finding is that it is the result of temporal uncertainty. If the observer assumes that the target is temporally near the cue, he or she will be more likely to respond with a digit that is perceived as temporally close to the cue than with one that is perceived as far from the cue. This bias would lead to the same results for stimuli with cues in both streams as for those with cues only in the same stream, which is what Klein and Dick reported. If this explanation is correct, then the results of Klein and Dick represent what Prinzmetal et al. (2005) termed "channel selection"- that is, the choice of location to which to respond. The results would not be an indication of "channel enhancement" - a change in the perceptual representation.

\section{Method}

Experiment 4 was a replication of the experiment of Klein and Dick (2002), but with some changes. Most notably, each observer was run with the stimuli in a different random order. In addition, feedback was given: The computer made an audible beep when participants erred. The purpose of the feedback was to make sure that the observers would not develop a bias to respond with a digit that they perceived to be in close spatial/temporal proximity to the cue. Feedback was also given at the end of each block, indicating the percentage correct responses in the respective block (in the original study there was no feedback).
There were some other differences between Experiment 3 (an exact replication of Klein and Dick's, 2002, experiment) and Experiment 4 . In the present experiment, we tested only same-stream-cued and opposite-stream-cued conditions; thus, there were 14 unique conditions instead of 28 . We wanted to have as powerful an experiment as possible and therefore ran only the theoretically important conditions. In Klein and Dick's study, there was one block of 28 practice trials followed by a single block of 448 test trials. In Experiment 4, there were two blocks of 14 practice trials each, and data were collected in six blocks of 56 trials each. Thus, we were able to collect more data (from 336 trials) on the two critical conditions than Klein and Dick could (from 224 trials). We have found that breaking the experiment into blocks keeps observers more motivated. Furthermore, by breaking the experiment into blocks that included all conditions, we were able to assess the improvement in performance over blocks. In Klein and Dick's study, participants initiated each trial with the space bar, whereas in the replication each trial began $1.5 \mathrm{sec}$ after the response from the previous trial. In the present experiment, each of the 14 conditions was run an equal number of times within each block, and a different random order was used for each block and each participant. In all other respects, such as display timing, stimulus size, font, and gray values, the experiment was identical to Experiment 3 (and to the experiment of Klein and Dick). As in Klein and Dick's experiment, there were 40 participants.

\section{Results and Discussion}

The results are shown in Figure 3 (bottom panel). The effect of cue validity was not significant $[F(1,39)=1.99$, $p>.05]$, nor was the interaction of validity and target frame $[F(6,234)=1.42, p>.05]$. Thus, we did not obtain the results of Klein and Dick (2002). Note that the means tend in the direction opposite those of Klein and Dick (Figure 3, top panel). Furthermore, we doubt that the lack of significant results was a consequence of a lack of power, since we ran twice as many observers as we had in Experiment 3.

There were, however, two significant effects. Target frame was significant $[F(6,234)=3.42, p<.05]$. The observers were more accurate when the target appeared in the most central frames (Frames 8 and 9). This might be an expectation effect, because the target occurred equally often in each of Frames 6-12, and therefore Frames 8 and 9 were the "average" target positions. Performance improved significantly over the six blocks $[F(5,195)=5.82$, $p<.01]$, but this factor did not significantly interact with any other variable. The average percentages correct for the first and last blocks were $80.3 \%$ and $86.7 \%$, respectively.

There are three possible reasons for the lack of cuing effect in Experiment 4 in comparison with Experiment 3 (and with Klein \& Dick, 2002). First, it might be that some incidental change (e.g., breaking up the task into blocks) caused the difference. If that is the case, the original finding does not generalize very well. More likely causes probably involve one of the two substantive changes that we made: giving feedback and randomizing the order of presentation. If the critical factor is giving feedback so that observers are not biased to respond to a digit that they perceive in close spatial/temporal proximity to the target, then the original results (and the results of Experiment 3) reflect channel selection (an effect on which location to respond to). If the critical difference is related to the order of presentation, then the original results reflect only an un- 
fortunate computer programming error. The fact that we have not been able to resolve this issue does not affect our main conclusion: The results of Klein and Dick cannot be taken as definitive evidence that a noninformative spatial cue will enhance accuracy in an RSVP task.

It is worth noting that even if we had replicated Klein and Dick's (2002) results in Experiment 4, there are other problems with their experiment that would have lent themselves to alternative explanations. We have already mentioned that Klein and Dick did not monitor eye movements (and of course, neither did we). It is quite clear from Experiments 1 and 2 that eye movements can inadvertently contaminate covert attention studies. A second factor is that their cues were, in fact, informative in that they provided information about when the target digit would occur. Although their cues were not spatially informative, it is possible that the fact that they were temporally informative may have biased observers to attend to them. (See Pashler, 1988, for an example in which informativeness in one domain can influence search in another domain.) In summary, when we used a random order of presentation and provided feedback, we did not replicate Klein and Dick's findings. However, even if we had, there still would have been issues about how to interpret their results.

\section{GENERAL DISCUSSION}

The motivation for this study came from Prinzmetal et al. (2005). They found that although voluntary attention and involuntary attention both affect performance in experiments designed around RT, only voluntary attention affects performance in experiments designed around accuracy. Note that, unlike with involuntary attention, with voluntary attention there is little doubt that predictive cues can affect accuracy (see, e.g., Luck et al., 1996; Smith, 2000). These studies clearly meet all of the criteria and show that, at moderate cue-target intervals, participants are more accurate with the target in the cued location than with the target in an uncued location.

Despite a clear effect on accuracy with voluntary attention, in seven experiments with involuntary attention Prinzmetal et al. (2005) found no effect on accuracy. The results of Dufour (1999) and of Klein and Dick (2002) seem to be exceptions to the rule. In an attempt to understand the critical difference that may lead to an effect on accuracy, we replicated the experiments of Dufour (with auditory cues) and Klein and Dick (with RSVP). In both cases, we found that the results could be accounted for by factors other than covert attention. In the case of Dufour's experiment, the results seem to have been due to eye movements. The results of Klein and Dick may have been due to spatial/temporal uncertainty or to trial order.

In other studies, claims of finding effects of noninformative cues on accuracy have been made (e.g., Cheal \& Chastain, 1999; Handy et al., 1999; Henderson \& Macquistan, 1993; Horstmann, 2002; McDonald et al., 2000). However, Prinzmetal et al. (2005) argued that these studies have not adequately met four criteria needed to convincingly demonstrate an effect, in an accuracy experiment, of automatic (noninformative) cues that is related to the quality of the perceptual representation. The first criterion is that eye movements be controlled. Second, the experiment must truly be an accuracy experiment, not a hybrid accuracy-RT experiment. Observers should be told to take their time and be as accurate as possible, rather than be encouraged to be "fast and accurate." They should not be given a deadline for responding. Third, there should be no location uncertainty. Observers should be nearly 100\% accurate at knowing where the stimulus was. Finally, cue validity should not be confounded with any other factor. In Klein and Dick's (2002) study, same-stream targets appearing immediately after the cue (valid trials) occurred on average later in the experiment than other-stream targets (invalid trials).

There may be exceptions to the generalization that noninformative spatial cues do not affect performance in accuracy experiments. The task of future research is not just to discover exceptions but to help understand the critical factors that would lead to such an effect.

Prinzmetal et al. (2005) claimed that Wundt (1902) was incorrect; voluntary attention affects the perceptual representation, but involuntary attention does not. Because of the problems described here, Dufour's (1999) and Klein and Dick's (2002) results cannot be considered definitive in resolving this issue.

\section{REFERENCES}

Banks, W. P., Larson, D. W., \& Prinzmetal, W. (1979). Asymmetry of visual interference. Perception \& Psychophysics, 25, 447-456.

Bouma, H. (1970). Interaction effects in parafoveal letter recognition. Nature, 226, 177-178.

CAmeron, E. L. (1995). Factors determining the latency of saccadic eye movements (Doctoral dissertation, University of Rochester). Dissertation Abstracts International, 56, 2909.

Cheal, M., \& Chastain, G. (1999). Inhibition of return: Support for generality of the phenomenon. Journal of General Psychology, 126, 375-390.

Dufour, A. (1999). Importance of attentional mechanisms in audiovisual links. Experimental Brain Research, 126, 215-222.

Fischer, B., \& RAMSPERGER, E. (1984). Human express saccades: Extremely short reaction times of goal directed eye movements. Experimental Brain Research, 57, 191-195.

Fischer, B., \& WeBER, H. (1993). Express saccades and visual attention. Behavioral \& Brain Sciences, 16, 553-610.

Fischer, B., \& WEBER, H. (1998). Effects of pre-cues on voluntary and reflexive saccade generation: I. Anti-cues for pro-saccades. Experimental Brain Research, 120, 403-416.

Gezeck, S., Fischer, B., \& Timmer, J. (1997). Saccadic reaction times: A statistical analysis of multimodal distributions. Vision Research, 37, 2119-2131.

Handy, T. C., JHA, A. P., \& Mangun, G. R. (1999). Promoting novelty in vision: Inhibition of return modulates perceptual-level processing. Psychological Science, 10, 157-161.

Harwood, M., Madelain, L., Krauzlis, R., \& Wallman, J. (2003, May). Spatial scale of attention strongly modulates saccade latency, but not by modulating stimulus saliency. Poster presented at the annual meeting of the Vision Sciences Society, Sarasota, FL.

Helmholtz, H. von (1896). Handbuch der physiologischen Optik. Leipzig: Voss.

Henderson, J. M., \& Macquistan, A. D. (1993). The spatial distribution of attention following an exogenous cue. Perception \& Psychophysics, 53, 221-230.

Horstmann, G. (2002). Evidence for attentional capture by a surprising color singleton in visual search. Psychological Science, 13, 499-505. 
James, W. (1890). The principles of psychology. New York: Holt.

JonidES, J. (1976, November). Voluntary vs. reflexive control of the mind's eye's movement. Paper presented at the annual meeting of the Psychonomic Society, St. Louis.

Jonides, J. (1980). Towards a model of the mind's eye's movement. $\mathrm{Ca}$ nadian Journal of Psychology, 34, 103-112.

JoNIDES, J. (1981). Voluntary versus automatic control over the mind's eye's movement. In J. B. Long \& A. D. Baddeley (Eds.), Attention and performance $I X$ (pp. 187-204). Hillsdale, NJ: Erlbaum.

Juola, J. F., Koshino, H., \& Warner, C. B. (1995). Tradeoffs between attentional effects of spatial cues and abrupt onsets. Perception \& Psychophysics, 57, 333-342

JÜTTNER, M., \& WoLF, W. (1994). Stimulus uncertainty and the occurrence of human express saccades. In G. d'Ydewalle \& J. van Rensbergen (Eds.), Visual and oculomotor functions: Advances in eye movement research (pp. 69-80). Amsterdam: North-Holland.

KLeIN, R. M., \& Dick, B. (2002). Temporal dynamics of reflexive attention shifts: A dual-stream serial visual presentation. Psychological Science, 13, 176-179.

KLeIN, R. M., \& SHORE, D. I. (2000). Relations among modes of visual orienting. In S. Monsell \& J. Driver (Eds.), Control of cognitive processes: Attention and performance XVIII (pp. 195-208). Cambridge, MA: MIT Press.

Loftus, G. R., \& Masson, M. E. J. (1994). Using confidence intervals in within-subject designs. Psychonomic Bulletin \& Review, 1, 476-490

Luck, S. J., Hillyard, S. A., Mouloua, M., \& Hawkins, H. L. (1996). Mechanisms of visual-spatial attention: Resource allocation or uncertainty reduction? Journal of Experimental Psychology: Human Perception \& Performance, 22, 725-737.

Luck, S. J., \& Thomas, S. J. (1999). What variety of attention is automatically captured by peripheral cues? Perception \& Psychophysics, 61, 1424-1435.

Madelain, L., Harwood, M., Krauzlis, R. J., \& Wallman, J. (2004, May). Spatial scale of attention influences saccade latencies. Poster presented at the annual meeting of the Vision Sciences Society, Sarasota, FL.

McDonald, J. J., Teder-Sälejërvi, W. A., \& Hillyard, S. A. (2000). Involuntary orienting to sound improves visual perception. Nature, 407, 906-908.

MülleR, H. J., \& Rabbitt, P. M. A. (1989). Reflexive and voluntary orienting of visual attention: Time course of activation and resistance to interruption. Journal of Experimental Psychology: Human Perception \& Performance, 15, 315-330.

PASHLER, H. (1988). Cross-dimensional interaction and texture segregation. Perception \& Psychophysics, 43, 307-318.

Pelli, D. G., Palomares, M., \& MajaJ, N. J. (2004). Crowding is unlike ordinary masking: Distinguishing feature integration from detection. Journal of Vision, 4, 1136-1169.

Posner, M. I. (1978). Chronometric explorations of mind. Hillsdale, NJ: Erlbaum.

Posner, M. I. (1980). Orienting of attention. Quarterly Journal of Experimental Psychology, 32, 3-25.

Posner, M. I., \& CoHEn, Y. (1984). Components of visual orienting. In H. Bouma \& D. G. Bouwhuis (Eds.), Attention and performance X; Control of language processes (pp. 531-555). Hillsdale, NJ: Erlbaum.

Posner, M. I., Cohen, Y., \& Rafal, R. D. (1982). Neural systems control of spatial orienting. Philosophical Transactions of the Royal Society of London: Series B, 298, 187-198.

Posner, M. I., SNyder, C. R. R., \& Davidson, B. J. (1980). Attention and the detection of signals. Journal of Experimental Psychology: General, 109, 160-174.

Prinzmetal, W., McCool, C., \& Park, S. (2005). Attention: Reaction time and accuracy reveal different mechanisms. Journal of Experimental Psychology: General, 134, 73-92.

Prinzmetal, W., Nwachuku, I., Bodanski, L., Blumenfeld, L., \& Shimizu, N. (1997). The phenomenology of attention: Part 2. Brightness and contrast. Consciousness \& Cognition, 6, 372-412.

Prinzmetal, W., Presti, D. E., \& Posner, M. I. (1986). Does attention affect visual feature integration? Journal of Experimental Psychology: Human Perception \& Performance, 12, 361-369.

Rafal, R. D., Calabresi, P. A., Brennan, C. W., \& Sciolto, T. K. (1989). Saccade preparation inhibits reorienting to recently attended locations. Journal of Experimental Psychology: Human Perception \& Performance, 15, 673-685.

RichaRD, C. M., Wright, R. D., \& WARD, L. M. (2003). Goal-driven modulation of stimulus-driven attentional capture in multiple-cue displays. Perception \& Psychophysics, 65, 939-955.

Sekuler, R., \& Blake, R. (1985). Perception. New York: Knopf.

ShIU, L. P., \& PAShler, H. (1994). Negligible effect of spatial precuing on identification of single digits. Journal of Experimental Psychology: Human Perception \& Performance, 20, 1037-1054.

Smith, P. L. (2000). Attention and luminance detection: Effects of cues, masks, and pedestals. Journal of Experimental Psychology: Human Perception \& Performance, 26, 1401-1420.

SPENCE, C. [J.] (2001). Crossmodal attentional capture: A controversy resolved? In C. L. Folk \& B. S. Gibson (Eds.), Attraction, distraction and action: Multiple perspectives on attentional capture (pp. 231262). Amsterdam: Elsevier, North-Holland.

Spence, C. J., \& Driver, J. (1994). Covert spatial orienting in audition: Exogenous and endogenous mechanisms. Journal of Experimental Psychology: Human Perception \& Performance, 20, 555-574.

Treisman, A., \& Gelade, G. (1980). A feature-integration theory of attention. Cognitive Psychology, 12, 97-136.

Warner, C. B., Juola, J. F., \& Koshino, H. (1990). Voluntary allocation versus automatic capture of visual attention. Perception \& Psychophysics, 48, 243-251.

Weber, H., Biscaldi, M., \& Fischer, B. (1995). Intertrial effects of randomization on saccadic reaction times in human observers. Vision Research, 35, 2615-2642.

WundT, W. (1902). Outlines of psychology (C. H. Judd, Trans.). Leipzig: Engelmann.

Wundt, W. (1912). An introduction to psychology (R. Pinter, Trans.). London: George Allen.

\section{NOTES}

1. Channel selection can influence accuracy, as discussed below, in circumstances in which there is location uncertainty - that is, observers are uncertain about which location contained the target (see, e.g., Luck \& Thomas, 1999; Shiu \& Pashler, 1994). However, this effect is not caused by an enhancement of the perceptual representation but rather by a bias to respond on the basis of information in the cued location. In the accuracy experiments of Prinzmetal et al. (2005), observers were close to $100 \%$ correct in knowing where the target stimulus was, but they were only $70 \%-85 \%$ correct in knowing the target's identity.

2. Prinzmetal et al. (2005) found that this arrangement of the speakers led to reliable cuing effects with noninformative cues in experiments designed around RT. Thus, we believe that the tones were adequate to elicit an automatic effect. Clear separation between auditory cues (provided by the speakers near the observer) may enhance auditory cuing effects (Spence \& Driver, 1994).

3. In Experiment 1, we observed eye movements on $75 \%$ of the trials. However, because of the way we monitored eye movements, we cannot ascertain the timing of the movement exactly. Some of these might have occurred after the stimulus was erased. Nevertheless, when eye movements were not actively suppressed in this experiment, they were frequent.

4. Saccadic latencies that average $100-150 \mathrm{msec}$ have been found both with the gap procedure, which is related to "express saccades" (see Fischer \& Weber, 1993), and without the gap procedure.

5 . The situation was completely different with predictive cues. In an experiment with predictive cues (which control voluntary attention), observers were significantly more accurate on valid than on invalid trials.

6. Subsequent to conducting the analysis presented below, we were told that 5 of their 40 participants were tested in a different order, which was not provided to us. Klein and Dick (2002) did not intend to use fixed orders; this occurred due to a communication failure between the experimenter and the programmer (R. Klein, personal communication, November 2002).

(Manuscript received April 29, 2004; revision accepted for publication February 15, 2005.) 